\title{
Ovarian Low Grade Endometrioid Stromal Sarcoma
}

National Cancer Institute

\section{Source}

National Cancer Institute. Ovarian Low Grade Endometrioid Stromal Sarcoma. NCI

Thesaurus. Code C40063.

A rare invasive mesenchymal tumor that arises from the ovary. It is characterized by the absence of marked pleomorphism and nuclear atypia in the neoplastic mesenchymal cells. It may have a favorable clinical outcome. 